\title{
An exploratory investigation examining male and female students' initial impressions and expectancies of lecturers
}

The aim of this study was to examine the informational cues that male and female students perceive to be influential when developing initial impressions and expectancies of a lecturer. University students $(n=752)$ rated the extent to which 30 informational cues influence their initial perceptions of a lecturer. Following exploratory factor analysis (EFA), a five-factor model (i.e., appearance (APP), accessories (ACC), third party reports (TPR), communication skills (CS), nationality/ethnicity (NE)) was extracted for male students and a five-factor model (i.e., ACC, TPR, APP, interpersonal skills (IPS), engagement (ENG)) extracted for female students. Inspection of mean scores identified that male students rated CS (e.g., clarity of voice) and TPR (e.g., qualifications) and female students IPS (e.g., control of class), ENG (e.g., eye contact) and TPR to be influential factors in forming initial impressions and expectancies of a lecturer. The findings further identify the potential for expectancy effects within student-lecturer interactions.

Keywords: impression formation; information cues; person perception; teaching

\section{Introduction}

The extant research literature (e.g., Babad, Inbar, and Rosenthal 1982; Jussim 1986; Jussim and Harber 2005) suggests that expectancy effects are rife in teaching. Unfortunately, however, there has been little research conducted to examine how students and/or lecturers form their beliefs and expectancies of one another. Yet, one study which did explicitly examine the cues perceived to be most influential during the initial formation of interpersonal impressions and expectancies was conducted by Birch, Batten, Manley, and Smith (2012). 
These researchers sought to identify the sources of information that sports students perceive to be influential when forming initial impressions of a lecturer. Exploratory factor analysis (EFA) revealed a five-factor model consisting of informational sources related to ‘appearance’ (e.g., attractiveness), ‘accessories’ (e.g., items of jewellery), ‘third party reports’ (e.g., qualifications), ‘communication skills’ (e.g., speed of speech), and 'nationality/ethnicity'. Mean data also showed that dynamic cues (i.e., communication skills) and third party reports were deemed by sports students to be more influential in their impressions and expectancies of a lecturer than static (i.e., appearance, accessories and nationality/ethnicity) cues. Such findings provide some support for Cook’s (1971) suggestion that static and dynamic cues are the two main sources of information that people use to form initial impressions and expectancies of others. However, Birch et al. (2012) also observed dynamic cues and third party reports to be two distinct sources of information, which would appear to suggest that dual-classifications of informational cues may warrant revision when applied within the context of sports students’ impression formation.

There is also some evidence to suggest that students’ perceptions and expectancies of lecturers may to some extent be influenced by gender stereotypes. For example, Kaschak (1978) and Lombardo and Tocci (1979) found that male students rated male professors more positively than female professors, while female students showed no professor gender bias. Basow and Silberg (1987) also reported that male students rated female professors significantly less positively than male professors. On the other hand, Bennett (1982), Elmore and LaPointe $(1974,1975)$ and Feldman (1992) failed to find any real evidence of gender bias in perceptions of teaching effectiveness. More recently, Centra and Gaubatz (2000) found that when students rated the same lecturer (i.e., when students were in the same class), female lecturers received higher ratings from female students, while no significant differences were observed in the ratings of male lecturers from both male and female 
students. Thus, it would seem beneficial to further examine the informational cues that are perceived by both male and female students to be influential in the formation of their beliefs and expectancies regarding lecturing staff. Indeed, these cues could provide a catalyst for the development of strategies aimed at (1) alleviating the potential negative impact of expectancies (e.g., student drop-out) and (2) maximising the potential benefits (e.g., enhanced student engagement). Consequently, the aim of the present study was to directly build upon the work of Birch et al. (2012) by examining the observable cues that both male and female sports students perceive to be influential when developing initial impressions and expectancies of a lecturer.

\section{Method}

\section{Participants}

The 452 participants recruited by Birch et al. (2012) were augmented with an additional 300 participants to create a total pool of 752 participants in the present study. The mean age of these participants was 20.16 years (SD = 3.13 years) and consisted of 449 men $(60 \%)$ and 303 women (40\%). Ninety-two per cent of participants $(n=688)$ were White-British, with the remaining 8\% consisting of Asian/Asian British - Indian $(n=2)$, Chinese $(n=2)$, other Asian background ( $n=3)$, Mixed - White and Black Caribbean $(n=9)$, Mixed - White and Asian ( $n=10)$, Black/Black British - African $(n=5)$, Black/Black British - Caribbean $(n=$ 5), White - Irish ( $n=9)$, Other White background ( $n=12)$, Other Mixed background $(n=5)$ and other Ethnic background ( $n=1)$ participants. Eighty-seven per cent of participants were studying on single honours degree programmes $(n=657)$, with the remaining $13 \%$ of participants studying on combined honours degree programmes $(n=95)$. All participants were studying for sports-related degrees. Ninety-five per cent of participants were full-time students $(n=712)$, with the remaining $5 \%$ studying on a part-time basis $(n=40)$. The 
majority of participants were in their first $(n=267)$ or second year $(n=311)$ of study; which equated to $36 \%$ and $41 \%$, respectively. The remaining $23 \%$ of participants were in their third ( $n=169)$ or fourth $(n=5)$ year of study. All participants were recruited from five public Universities in the United Kingdom (four from Southern England and one from South-East Scotland); four of which could be considered new (post 1992) Universities and one a pre1992 University. However, within all of the Universities examined, sports courses were amongst the top ten recruiters in terms of student numbers.

\section{Measures}

\section{Student Impression Formation Questionnaire}

The Student Impression Formation Questionnaire (SIFQ; Birch et al. 2012) was used to determine the impression cues students perceive to be most important when forming initial impressions and expectancies of a lecturer. Here, participants were presented with a pool of 30 constructs and then asked to read the following sentence, completing it by inserting each of the listed constructs in turn: 'When forming an initial impression of a lecturer, is a major source of information that influences my impressions.' Participants were then required to rate their agreement with each of the sentences they had formulated using the pool of constructs. Each item was listed and evaluated using a 7-point Likert scale ranging from 1 (very strongly disagree) to 7 (very strongly agree). The SIFQ also afforded participants with the opportunity to suggest for any further sources of information (not listed) that they may use to form initial impressions and expectancies of a lecturer.

\section{Procedure}

Participants were recruited over a period of approximately one year. All participants were enrolled on a sports-related University degree programme at the time of data collection. The 
experimental protocol was explained to the participants and written informed consent obtained. The SIFQ, which took around 10-20 minutes to complete, was completed in the presence of at least one of the authors to ensure that any questions from the participants could be answered. Ethical approval was obtained from the University prior to data collection.

\section{Data Analysis}

Mean scores for items on the SIFQ were calculated in an attempt to identify the sources of information that male and female sports students deem most influential when forming initial impressions and expectancies of a lecturer. An EFA of ratings obtained from the SIFQ was also conducted in order to determine the collective factors associated with the cues male and female sports students reported to be influential in expectancy formation. Principalcomponent analysis (PCA) was the extraction method used and the varimax method of rotation was implemented for both genders. Conceptual analysis was also used to categorise the additional sources of information that male and female sports students may utilise to form initial impressions and expectancies of a lecturer.

\section{Results}

\section{Descriptive Statistics}

The primary aim of the current investigation was to identify the information cues that influence the formation of male and female sports students' initial impressions and expectancies of a lecturer. Mean scores and standard deviations for the items included in the SIFQ for male students are displayed in Table 1 and are ranked in descending order of their means. Findings revealed that clarity of voice, control of class, level of preparation, body language/gestures and speed of speech were highly rated by male sports students as information cues that influence their initial formation of impressions and expectancies of a 
lecturer. In contrast, wearing of glasses, ethnicity, gender, nationality and facial hair received low ratings from male students' with regards to the perceived importance of these items when forming initial impressions and expectancies of a lecturer.

\section{INSERT TABLE 1 HERE}

Mean scores and standard deviations for the items included in the SIFQ for female sports students are displayed in Table 2 and are also ranked in descending order of their means. Similar to male students, findings revealed that clarity of voice, level of preparation, control of class, speed of speech and body language/gestures were highly rated by female students as information cues that influence their initial formation of impressions and expectancies of a lecturer. Conversely, wearing of glasses, ethnicity, gender, nationality and items of jewellery received low ratings from female sports students' with regards to the perceived importance of these items when forming initial impressions and expectancies of a lecturer.

\section{INSERT TABLE 2 HERE}

\section{Data Reduction}

In order to ensure that the assumptions of EFA were satisfied, sampling adequacy was tested via the Kaiser-Meyer-Olkin (KMO) statistic. According to Garson (2006), KMO values must exceed 0.60 to proceed with EFA. The KMO statistic regarding the SIFQ was 0.822 for male and 0.815 for female students. Pearson Product Moment Correlation Coefficients also identified no evidence of multicollinearity (as all were below 0.80; Stevens 1996) for both genders. 
For male sports students, PCA using the Anderson-Rubin method with orthogonal (varimax) rotation identified nine factors with eigenvalues $>1.0$. However, since Parallel Analysis (PA) has been proposed as a more reliable and accurate method of identifying the number of factors within a data-set (Franklin, Gibson, Robertson, Pohlmann, and Fralish 1995), only those component PCA eigenvalues which were greater than their relative component PA eigenvalues were retained.

Following the use of PA, a five-factor model of information sources was therefore suggested for the male students. This model explained approximately $46 \%$ of the cumulative variance. In line with the procedure utilised by Birch et al. (2012), only items with a factor loading above 0.50 were included within the PCA. The identified factors, total variance explained, associated variables and rotated factor loadings are displayed in Table 3.

Examination of factor loadings identified that physique/body type, age, gender and attractiveness should be collated to form one factor. This factor was labelled 'appearance' (APP). The sources of information within the second factor comprised of facial hair, items of jewellery, tattoos/piercings, wearing of glasses and hair style. This factor was labelled 'accessories' (ACC). The third factor was labelled 'third party reports' (TPR) and comprised of five variables: qualifications, teaching experience, consultancy/applied experience, number of research publications and reputation. The fourth factor, 'communication skills' (CS), was comprised of language (e.g., simple/complex), tone of voice, speed of speech and clarity of voice. The final factor comprised of nationality, ethnicity and accent of voice and was labelled 'nationality/ethnicity' (NE). Inspection of variable means revealed that male sports students deem CS and TPR to be more influential than APP, NE and ACC in determining their initial impressions and expectancies of a lecturer. 
For female students, PCA using the Anderson-Rubin method with orthogonal (varimax) rotation identified eight factors with eigenvalues $>1.0$. However, following the use of PA, a five-factor model of information sources was also suggested. This model explained approximately $50 \%$ of the cumulative variance. In line with the procedure utilised by Birch et al. (2012), only items with a factor loading above 0.50 were included within the PCA. The identified factors, total variance explained, associated variables and rotated factor loadings are displayed in Table 4.

Examination of factor loadings identified that facial hair, items of jewellery, tattoos/piercings and wearing of glasses should be collated to form one factor. This factor was labelled 'accessories' (ACC). The sources of information within the second factor comprised of qualifications, teaching experience, number of research publications, consultancy/applied experience and reputation. This factor was labelled 'third party reports' (TPR). The third factor was labelled ‘appearance’ (APP) and comprised of three variables: attractiveness, physique/body type and hair style. The fourth factor, 'interpersonal skills' (IPS), was comprised of speed of speech, odour (e.g., body/breath), control of class, level of preparation and clarity of voice. The final factor comprised of eye contact, tone of voice, posture and facial expressions and was labelled 'engagement' (ENG). Inspection of variable means revealed that female sports students deem IPS, ENG and TPR to be more influential than APP and ACC in determining their initial impressions and expectancies of a lecturer.

\author{
INSERT TABLE 4 HERE
}

\title{
Conceptual Analysis
}


In addition to the factors assessed as part of the SIFQ, 17\% of male sports students $(n=77)$ and $26 \%$ of female sports students $(n=80)$ provided additional information cues that they considered influential when forming initial impressions and expectancies of a lecturer. In accordance with the guidelines proposed by Krippendorff (1980), conceptual analysis was performed on this data. This enabled the data to be coded into meaningful units of information so that specific characteristics of the verbatim could be categorised in relation to the research aims. Four main themes were identified that were common across both male and female students: ability to engage the class/sense of humour, approachability, enthusiasm to teach, and confidence.

'Ability to engage the class/sense of humour' ( $n=10$ males, $n=10$ females) was the most prevalent theme identified following the conceptual analysis of both male and female sports students’ suggestions. Male and female student responses seemed to suggest that a lecturer's ability to engage the class through means of humour was valued to facilitate a comfortable learning environment. The second theme extracted from the conceptual analysis was labelled 'approachability' ( $n=6$ males, $n=8$ females). Male and female sports student responses from this category seemed to suggest that approachability would help build stronger relationships with students in that they would feel more confident in asking questions of their lecturer. The third theme extracted from the conceptual analysis was labelled 'enthusiasm to teach' ( $n=5$ males, $n=5$ females). Male and female sports student responses from this category seemed to suggest that enthusiasm to teach is associated with an informative teaching style which could aid student learning. The fourth theme extracted was classified as ‘confidence’ ( $n=5$ males, $n=4$ females). Male and female sports student responses from this theme seemed to suggest that students value confident lecturers in that this characteristic exudes competence. 
However, two additional themes were also identified following the conceptual analysis of male and female sports student's suggestions: quality of explanations, and friendliness. The first theme that was extracted was labelled 'quality of explanations' and was identified by male students $(n=7)$ only. Male sports student responses from this category seemed to suggest that the use of multiple teaching methods helps with understanding complex material and helps to maintain concentration and engagement. The second theme extracted was labelled 'friendliness' and was identified by female students $(n=4)$ only. Female sports student responses from this category seemed to suggest that friendliness would help to further develop relationships with students and would facilitate a comfortable learning environment.

Thus, the additional information identified by the conceptual analysis provides further support for the mean scores for items of the SIFQ and the two five-factor models extracted via EFA. More specifically, all of the additional themes identified above could be categorised as either communication or interpersonal skill cues. However, there was no indication from the male and/or female sports student self-reports that the SIFQ was missing static cues that might otherwise be influential when forming initial impressions and expectancies of a lecturer. Therefore, it might be argued that the conceptual analysis reinforces the results of the mean ratings identified by the SIFQ and the EFA in that both male and female students appear to regard dynamic cues and TPR as more influential than static cues when impressions and expectancies of a lecturer are initially formed.

\section{Discussion}

The aim of the present study was to directly build upon the work of Birch et al. (2012) by examining the cues that male and female sports students perceived to be influential when forming initial impressions and expectancies of a lecturer. Mean ratings identified by the 
SIFQ suggested that clarity of voice, control of class, level of preparation, speed of speech, and body language/gestures were the most influential cues in shaping both male and female students’ initial impressions and expectancies of a lecturer. In contrast, wearing glasses, ethnicity, gender and nationality received the lowest mean ratings from both male and female students, suggesting that these cues are less influential in forming initial impressions and expectancies of a lecturer. In line with the contentions of Cook (1971) and Birch et al. (2012), the findings of the present study would therefore appear to suggest that both male and female sports students are more likely to use dynamic behavioural cues than static sources of information in the formation of their beliefs and expectancies regarding lecturing staff.

EFA of student ratings also yielded two five-factor models regarding the informational cues that male and female sports students attend to when forming initial impressions and expectancies of a lecturer. The five components extracted for male sports students' were labelled APP, ACC, TPR, CS and NE, whereas the five components extracted for female sports students' were labelled ACC, TPR, APP, IPS and ENG. The APP, ACC and NE factors for male students, and the ACC and APP factors for female students, consist of static cues (e.g., gender), meaning that not only are they more stable over time, but that they are also generally uncontrollable. On the other hand, the TPR and CS factors for male students, and the TPR, IPS and ENG factors for female students, encapsulate dynamic cues (e.g., speed of speech) that reflect episodic behaviours which are more malleable and under the control of the target.

However, there were also some notable differences between the two male and female five-factor models, as well as the factor structure identified by Birch et al. (2012). More specifically, within the male ACC factor, hair style also loaded as an additional source of information that sports students may use during initial impression formation. This differs to both the female ACC factor and the ‘accessories' factor identified by Birch et al. (2012), 
where only facial hair, items of jewellery, tattoos/piercings and wearing of glasses items loaded. In addition, within the male NE factor, accent of voice also loaded as an additional source of information that sports students may use when forming initial impressions and expectancies of a lecturer. This differs to the 'nationality/ethnicity' factor identified by Birch et al. (2012), where only the nationality and ethnicity items loaded. This male factor solution also differs to the identified female factor solution in that the nationality and ethnicity items (or factor) did not load for female students in the present study at all. Instead, the CS factor for males in the present study, and the ‘communication skills' factor identified by Birch et al. (2012), would seem to have been further sub-divided and/or expanded upon in order to form the IPS and ENG factors for female sports students in the present study.

Nonetheless, the two aforementioned five-factor models both provide further support for Cook’s (1971) suggestion that static and dynamic cues are the two main sources of information that people use to form initial impressions and expectancies of others. However, the findings from the present study and those of Birch et al. (2012) would also appear to suggest that the static-dynamic categories are not as simple as first thought. Indeed, the observed EFA findings indicate that the static cues classification might be further sub-divided into a number of distinct categories. More specifically, it might be argued that the ACC, APP and NE factors for male sports students, and the ACC and APP factors for female sports students, are sub-categories of the broader static cues classification. However, and in contrast to both the work of Birch et al. (2012) and the factor solution for the male students, the EFA findings for the female students in the present study would seem to indicate that the dynamic cues classification might also be further sub-divided into two distinct categories. More specifically, it might be argued that the IPS and ENG factors for female sports students are sub-categories of the broader dynamic cues classification. In addition, TPR were also clearly identified within both the male and female five-factor models within the present study and by 
Birch et al. (2012). Such findings add further credence to the suggestion that dualclassifications of informational cues may need to be expanded when applied within the context of sports students' impression formation.

The conceptual analysis within the present study would also appear to suggest that both male and female students regard dynamic cues and TPR as more influential than static cues when forming initial impressions and expectancies of a lecturer. These results support the claims of previous research (e.g., Becker and Solomon 2005; Cook 1971; Horn, Lox, and Labrador 2001; Jussim 1993; Jussim, Coleman, and Lerch 1987; Manley, Greenlees, Graydon, Thelwell, Filby, and Smith 2008; Birch et al. 2012) in that dynamic behavioural cues seem to be the major determinant of a perceiver's impression formation. This would especially seem to be the case when it comes to female sports students. Indeed, mean ratings identified by the SIFQ and the EFAs in the present study would appear to suggest that dynamic behavioural cues are even more important for female students than male students and/or that female students place even less importance on static cues than do male students. However, whilst the four main conceptual analysis themes identified for both male and female sports students support the additional sources of information reported by Birch et al. (2012), there were again some subtle differences between male and female students here also. Indeed, although both male students and Birch et al. (2012) suggested 'quality of explanations' to be an additional source of information used during student impression formation, female students suggested that the perceived 'friendliness' of the lecturer might be more important than the quality of their explanations.

Although the present study did not explicitly examine teaching evaluations, the findings contained herein may also have some important implications regarding gendered perceptions of teacher effectiveness. Indeed, findings from the conceptual analysis in the present study would appear to suggest that the 'quality of explanations' and perceived levels 
of 'friendliness' displayed by lecturers are likely to impact their subsequent interactions with male and female sports students, respectively. Such findings provide partial support for the work of Kaschak (1978), Lombardo and Tocci (1979), and Centra and Gaubatz (2000), who also found that evaluations of lecturers may vary between the two genders. However, inspection of mean item scores from the SIFQ revealed that the information cues that male and female sports students deem to be most influential in expectancy formation are very similar. These findings lend support to the work of Bennett (1982), Elmore and LaPointe $(1974,1975)$ and Feldman (1992), and suggest that any such differences in perceived lecturer effectiveness between male and female sports students is likely to be minimal. Yet, given the somewhat conflicting results from the conceptual analysis and SIFQ within the present study, as well as the equivocal nature of previous research examining gendered perceptions of teaching evaluations, this line of research interest would seem to warrant further investigation.

However, there are also a number of other possible explanations for the conflicting results previously highlighted. First, because the SIFQ was a self-report instrument, specific judgement biases may have influenced the overall findings. Second, Manley et al. (2008) argued that self-report ratings might not accurately reflect the cues that students use when forming expectancies because it is possible that the students themselves might not be aware of their encoding of certain cues. Third, and arguably the main limitation of both the present study and that of Birch et al. (2012) was the researchers failure to account for the entire range of cues that sports students may utilise when forming an initial impression of a lecturer. For example, Becker and Solomon (2005) reported that psychological cues (e.g., confidence) may also influence impression formation. Future research may therefore need to consider the influence of psychological cues when investigating initial impression formation. In addition, because the participants in both the present study and that of Birch et al. (2012) represented a 
fairly homogenous sample, it was not possible to investigate the effect of cues such as age and ethnic background on the way in which sports students form initial impressions of a lecturer. This may be a fruitful avenue for future research that may inform specific teaching practices based on the demographics of the class. Given that most sports students and courses within the United Kingdom promote the use of active learning strategies (Peters, Jones, and Peters 2008), it is also perhaps somewhat unsurprising to see the students in the present study draw heavily on dynamic cues when forming initial impressions and expectancies of a lecturer. Thus, future researchers may also wish to replicate this study in a number of different subject areas.

\section{Conclusion}

The aim of this study was to examine the informational cues that male and female sports students perceive to be influential when developing initial impressions and expectancies of a lecturer. Mean ratings identified by the SIFQ suggested that clarity of voice, control of class, level of preparation, speed of speech, and body language/gestures were the most influential cues in shaping both male and female sports students' beliefs and expectancies regarding lecturing staff. Thus, the findings from the present study indicate that dynamic cues are rated by both male and female sports students as being highly influential sources of information when forming initial impressions and expectancies of a lecturer. EFA of student ratings also yielded two five-factor models regarding the informational cues that male and female sports students attend to when forming initial impressions and expectancies of a lecturer. The five components extracted for male students' were labelled APP, ACC, TPR, CS and NE, whereas the five components extracted for female students' were labelled ACC, TPR, APP, IPS and ENG. The conceptual analysis within the present study would also appear to suggest that both 
male and female sports students regard dynamic cues and TPR as more influential than static cues when forming initial impressions and expectancies of a lecturer.

In line with the suppositions of Birch et al. (2012), the results of this exploratory investigation would therefore appear to suggest that by developing strategies to convey appropriate dynamic behavioural cues (e.g., clear projection of voice) and TPR (e.g., incorporating consultancy/applied experience to inform teaching), lecturers' may be able to facilitate student-lecturer interaction. However, such strategies may also need to consider the relative importance placed on dynamic behavioural cues and TPR by both male and female sports students. Indeed, the results of present study would appear to suggest that dynamic behavioural cues may be even more important for female students than male students. The findings from the conceptual analysis would also appear to support the claims of Belenky, Clinchy, Goldberger, and Tartule (1986) in that female students maybe more receptive than male students to a teaching methodology that values connections, understanding and acceptance. Yet, the two identified five-factor models clearly warrant further investigation in order to determine their validity across various student populations. The potential impact of student expectancies on subsequent student-lecturer interaction also requires further empirical examination.

\section{Acknowledgements}

Appreciation is extended to those students and lecturers who enabled data collection to take place during their classes.

\section{References}

Babad, E.Y., J. Inbar and R. Rosenthal. 1982. Pygmalion, Galatea, and the Golem: 
Investigations of biased and unbiased teachers. Journal of Educational Psychology 74: $459-74$.

Basow, S.A., and N.T. Silberg. 1987. Student evaluations of college professors: Are female and male professors rated differently? Journal of Educational Psychology 79: 308-14.

Becker, A.J., and G.B. Solomon. 2005. Expectancy information and coach effectiveness in intercollegiate basketball. The Sport Psychologist 19: 251-66.

Belenky, M., B.M. Clinchy, N.R. Goldberger, and J.M. Tartule. 1986. Women’s ways of knowing: The development of self, mind and voice. New York: Basic Books.

Bennett, S. 1982. Student perceptions and expectations for male and female instructors: Evidence relating to the question of gender bias in teaching evaluation. Journal of Educational Psychology 74: 170-9.

Birch, P.D.J., J. Batten, A.J. Manley, and M.J. Smith. 2012. An exploratory investigation examining the cues that students use to form initial impressions and expectancies of lecturers. Teaching in Higher Education.

Centra, J.A., and N.B. Gaubatz. 2000. Is there gender bias in student evaluations of teaching? The Journal of Higher Education 71: 17-33.

Cook, M. 1971. Interpersonal perception. Harmondsworth, UK: Penguin Education.

Elmore, P., and K. LaPointe. 1974. Effects of teacher sex, and student sex on the evaluation of college instructors. Journal of Educational Psychology 66: 386-9.

Elmore, P., and K. LaPointe. 1975. Effects of teacher sex, student sex, and teacher warmth on the evaluation of college instructors. Journal of Educational Psychology 67: 368-74.

Feldman, K.A. 1992. College students' views of male and female college teachers: Part 1Evidence from the social laboratory and experiments. Research in Higher Education 33: 317-51.

Franklin, S.B., D.J. Gibson, P.A. Robertson, J.T. Pohlmann, and J.S. Fralish. 1995. Parallel 
analysis: A method for determining significant principle components. Journal of Vegetation Science 6: 99-106.

Garson, G.D. 2006. Factor analysis. Statnotes: Topics in multivariate analysis. http://www2. chass.ncsu.edu/garson/pa765/factor.htm

Horn, T.S., C.L. Lox, and F. Labrador. 2001. The self-fulfilling prophecy theory: When coaches’ expectations become reality. In Applied sport psychology: Personal growth to peak performance, ed. J. Williams. Mountain View, CA: Mayfield.

Jussim, L. 1986. Self-fulfilling prophecies: A theoretical and integrative review. Psychological Review 93: 429-45.

Jussim, L. 1993. Accuracy in interpersonal expectations: A reflection-construction analysis of current and classic research. Journal of Personality 61: 637-68.

Jussim, L., L.M. Coleman, and L. Lerch. 1987. The nature of stereotypes: A comparison and integration of three theories. Journal of Personality and Social Psychology 52: 53646.

Jussim, L., and K.D. Harber. 2005. Teacher expectations and self-fulfilling prophecies: Knowns and unknowns, resolved and unresolved controversies. Personality and Social Psychology Review 9: 131-55.

Kaschak, E. 1978. Sex bias in student evaluations of college professors. Psychology of Women Quarterly 2: 235-43.

Krippendorff, K. 1980. Content analysis: An introduction to its methodology. Newbury Park, CA: Sage.

Lombardo, J., and M. Tocci. 1979. Attribution of positive and negative characteristics of instructors as a function of attractiveness and sex of instructor and sex of subject. Perceptual and Motor Skills 48: 491-4.

Manley, A.J., I. Greenlees, J. Graydon, R. Thelwell, W.C.D. Filby, and M.J. Smith. 2008. 
Initial impression formation of lecturers

Athletes' perceived use of information sources when forming initial impressions and expectations of a coach: An exploratory study. The Sport Psychologist 22: 73-89.

Peters, D., G. Jones, and J. Peters. 2008. Preferred 'learning styles’ in students studying sports-related programmes in higher education in the United Kingdom. Studies in Higher Education 33: 155-166.

Stevens, J. 1996. Applied multivariate statistics for the social sciences. Mahwah, NJ: Lawrence Erlbaum. 